\title{
羟基磷灰石微纳结构对蛋白吸附的影响
}

\author{
付亚康 $^{1,2}$, 周 雪 ${ }^{1}$, 肖东琴 ${ }^{1}$, 匙 峰 $^{1}$, 卢晓英 ${ }^{1}$, 翁 杰 $^{1}$
}

(1. 西南交通大学 材料先进技术教育部重点实验室, 成都 610031 ; 2. 雅安职业技术学院, 雅安 625000)

摘 要: 本研究采用水热反应法, 在不同浓度环已烷六羧酸 $\left(\mathrm{H}_{6} \mathrm{E}\right)$ 模板调控作用下, 合成了具有不同表面微纳结构的 羟基磷灰石(HAP)微粒，并采用 XRD、BET、FTIR 和 SEM 对其进行表征。对 HAP 微粒进行了牛血清白蛋白(BSA)、 纤维蛋白原(FN)和溶菌酶(LYS)的吸附及释放实验。结果表明: $\mathrm{H}_{6} \mathrm{E}$ 能够在 HAP 微粒表面构建微纳结构, 不同微纳 结构对不同蛋白质具有选择性吸附作用; 在 $\mathrm{H}_{6} \mathrm{E}$ 浓度为 $50 \mathrm{mmol} / \mathrm{L}$ 的合成条件下制备的中空结构 HAP 微粒(HAP50) 其载蛋白后体外释放具有明显的蛋白缓释性能。

关 键 词: 羟基磷灰石; 环己烷六羧酸; 微纳结构; 蛋白吸附

中图分类号: R318; TQ174 文献标识码: A

\section{Influence of Micro-nano Structure of Haydroxyapatite Particles on Protein Adsorption}

\author{
FU Ya-Kang ${ }^{1,2}$, ZHOU Xue ${ }^{1}$, XIAO Dong-Qin ${ }^{1}$, SHI Feng ${ }^{1}$, LU Xiao-Ying ${ }^{1}$, WENG Jie ${ }^{1}$
}

(1. Key Laboratory of Advanced Technologies of Materials, Ministry of Education, School of Materials Science and Engineering, Southwest Jiaotong University, Chengdu 610031, China; 2. Ya'an Vocational College, Ya'an 625000, China)

\begin{abstract}
Hydroxyapatite (HAP) particles were hydrothermally synthesized with the surface morphologies adjusted by cyclohexane-1, 2, 3, 4, 5, 6-hexacarboxylic acid $\left(\mathrm{H}_{6} \mathrm{E}\right)$ as the template. HAP particles were characterized by XRD, BET, SEM and FTIR. The protein adsorption-desorption behaviors of positively charged lysozyme (LYS), fibrinogen (FN) and negatively charged bovine serum albumin (BSA) on these HAP particles were examined. The results indicate that using $\mathrm{H}_{6} \mathrm{E}$ as a template to fabricate micro-nano structures on HAP particles through hydrothermal reaction is simple and controllable. HAP particles with micro-nano structures show selective protein adsorption-desorption properties for different proteins. The protein-loaded shell-like HAP particle (HAP50-protein) shows an excellent protein release behavior in vitro.
\end{abstract}

Key words: hydroxyapatite; $\mathrm{H}_{6} \mathrm{E}$; micro-nano structure; protein adsorption

生物材料植入体内后很快发生来自血液及其它 组织液中蛋白质在植入体表面的吸附，这层吸附的 蛋白质将直接影响细胞粘附、增殖和分化, 并最终 决定植入的成败 ${ }^{[1]}$ 。因此, 考察生物材料蛋白吸附行
为已经成为评价其生物相容性和生物活性的一种重 要方式 ${ }^{[2]}$ 。

羟基磷灰石 (hydroxyapatite, HAP)具有与人体 硬组织中相近的无机成分，其良好的生物相容性和

收稿日期: 2014-10-10; 收到修改稿日期: 2014-11-20

基金项目: 国家重点基础研究发展计划(2012CB933600); 国家自然科学基金(51172188); 四川省科技支撑计划(2010FZ0048); 四川省高校科研创新团队建设计划项目(14TD0050)

National Basic Research Program of China (2012CB933600); National Natural Science Foundation of China (51172188); Science and Technology Pillar Project of Sichuan (2010FZ0048); Research and Innovation Team Project of Sichuan for Central Universities(14TD0050)

作者简介: 付亚康(1988-), 男, 硕士研究生, 助教. E-mail: fykmail@163.com; 810667526@qq.com

通讯作者: 翁 杰, 教授. E-mail: jweng@swjtu.cn 
生物活性已被大量研究所证实 ${ }^{[3]}$, 因而被广泛地应 用于临床骨组织修复。近年来多孔 HAP 植入体显示 出优良的骨诱导性, 但其骨诱导机理还存在较多不 明之处 ${ }^{[4]}$ 。因此, 研究 HAP 表面蛋白吸附行为有助 于揭示其生物活性, 尤其是骨诱导性的本质, 并且 据此对其结构优化提供参考 ${ }^{[5]}$ 。

HAP 表面微纳结构、粗糙度 ${ }^{[6]}$ 、 孔隙率 ${ }^{[7]}$ 、孔 径大小 ${ }^{[8]}$ 以及比表面积 ${ }^{[9]}$ 等因素显著地影响其蛋白 吸附能力。不同离子 $\left(\mathrm{Zn}^{2+[10]}, \mathrm{CO}_{3}{ }^{2-[11]}\right)$ 在 HAP 中的 掺杂对 HAP 的蛋白吸附性能也具有重要影响。但是, 目前对其影响机理还不甚清楚, 有待进一步研究。

本研究旨在探讨 HAP 表面微纳结构对蛋白质 吸附的影响。采用水热-模板法制备具有不同表面微 纳结构的 HAP 微粒; 选用牛血清白蛋白(BSA)、纤 维蛋白原 (FN) 和溶菌酶(LYS)三种蛋白质, 研究其 在不同 HAP 微粒表面的蛋白吸附-释放行为, 并对 其机理进行初步的讨论。

\section{1 实验方法}

\section{1 材料的合成与制备}

借助模板分子 $\mathrm{H}_{6} \mathrm{E}$ 调控, 在水热条件下完成不 同 HAP 微粒的制备。主要原料为分析纯级的四水硝 酸钙 $\left(\mathrm{Ca}\left(\mathrm{NO}_{3}\right)_{2} \bullet 4 \mathrm{H}_{2} \mathrm{O}\right)$ 、十二水磷酸氢二钠 $\left(\mathrm{Na}_{2} \mathrm{HPO}_{4}\right.$ ・ $\left.12 \mathrm{H}_{2} \mathrm{O}\right)$, 尿素 $\left(\mathrm{CON}_{2} \mathrm{H}_{4}\right)$, 均购自成都市科龙化工试 剂厂。模板分子为分析纯级环已烷六羧酸(cyclohexane1, 2, 3, 4, 5, 6-hexacarboxylic acid, $\mathrm{H}_{6} \mathrm{E}$ ), 购自东京 化成工业株式会社。

在 $0.1 \mathrm{~mol} / \mathrm{L} \mathrm{Ca}\left(\mathrm{NO}_{3}\right)_{2} \bullet 4 \mathrm{H}_{2} \mathrm{O}$ 溶液中加入 $\mathrm{H}_{6} \mathrm{E}$, 使其浓度分别为 $0 、 0.5 、 1 、 5$ 和 $50 \mathrm{mmol} / \mathrm{L}$ 。然后 加入适量的 $\mathrm{Na}_{2} \mathrm{HPO}_{4} \cdot 12 \mathrm{H}_{2} \mathrm{O}$, 保持钙磷比为 1.67 : 1 。再加入 $0.56 \mathrm{~mol} / \mathrm{L} \mathrm{CON} \mathrm{H}_{4}$, 稀硝酸调节 $\mathrm{pH}$ 为 2.5 。将溶液转入反应釜, $150^{\circ} \mathrm{C}$ 下应 $5 \mathrm{~h}$ 。洗涤, 烘 干, 收集。不同浓度 $\mathrm{H}_{6} \mathrm{E}$ 条件下制备的 HAP 分别记 为 HAP0、HAP0.5、HAP1、HAP5 和 HAP50。

\section{2 蛋白质吸附实验}

$\mathrm{BSA} 、 \mathrm{FN}$ 和 LYS 均为分析纯, 购自上海华兰生 物制剂公司。将 BSA、FN 或 LYS 分别溶解于双蒸 水, 定容制备 $1 \mathrm{mg} / \mathrm{mL}$ 的蛋白溶液。

取不同 HAP 微粒各 $10 \mathrm{mg}$ 置于 $2 \mathrm{~mL}$ 离心管中, 分别加入 $1 \mathrm{~mL}$ 浓度 $1 \mathrm{mg} / \mathrm{mL}$ 的蛋白液。在 $37^{\circ} \mathrm{C}$ 、 $97 \mathrm{r} / \mathrm{min}$ 下吸附 $24 \mathrm{~h}$ 。用 $\mathrm{BCA}$ 试剂盒 $\left(\mathrm{BCA}^{\mathrm{TM}}\right.$ Protein Assay Kit, 南京建成生物制剂有限公司)测试上清 液中蛋白含量, 再根据质量守恒原理计算样品蛋白 吸附量。

\section{3 蛋白质释放实验}

将吸附了蛋白质的 HAP 微粒在 $\operatorname{PBS}(0.01 \mathrm{~mol} / \mathrm{L})$ 中进行体外蛋白释放。在 $37^{\circ} \mathrm{C} 、 97 \mathrm{r} / \mathrm{min}$ 条件下, 按 一定时间间隔取样，之后补入等体积 $\mathrm{PBS}$ 。用 $\mathrm{BCA}$ 法进行蛋白浓度测试，绘制释放曲线。

\section{4 仪器与测试}

分别采用 X射线衍射(XRD, Philips PW3040/60) 和扫描电子显微镜(SEM, QUANTA200)对制备微粒 相组成及表面形貌进行表征; 用粒度分布测试仪 (LA-920 HORIBA)和比表面积仪(BET, 3H- 2000BETA)对微粒的粒径和比表面积进行测试。用红外光谱 仪 (FTIR, Thermo Nicolet5700) 和倒置荧光显微镜 (CFM, IX51)对吸附蛋白质后的样品进行表征。

\section{2 结果与讨论}

\section{1 表面形貌}

不同浓度 $\mathrm{H}_{6} \mathrm{E}$ 合成条件下, 所获得的样品如图 1 所示。在合成过程中不添加 $\mathrm{H}_{6} \mathrm{E}$ 时, 产物为带状, 宽 $3 \mu \mathrm{m}$ 、长约 $30 \mu \mathrm{m}$ (图 1(a)); 当合成体系中 $\mathrm{H}_{6} \mathrm{E}$ 浓度为 $0.5 \mathrm{mmol} / \mathrm{L}$ 时, 产物呈短带状结构组装而成 的花瓣状, 短带状结构长约 $10 \mu \mathrm{m}$ (图 1(b)); 当 $\mathrm{H}_{6} \mathrm{E}$ 浓度为 $1 \mathrm{mmol} / \mathrm{L}$ 时, 产物为花梆菜状结构(图 1(c)); 可见在合成体系中 $\mathrm{H}_{6} \mathrm{E}$ 能够抑制带状结构沿其长轴 方向的生长。当合成体系中 $\mathrm{H}_{6} \mathrm{E}$ 浓度为 $5 \mathrm{mmol} / \mathrm{L}$ 时, 产 物为线球状(图 1(d)); 当 $\mathrm{H}_{6} \mathrm{E}$ 浓度为 $50 \mathrm{mmol} / \mathrm{L}$ 时, 产 物为中空结构 (图 1(e))。翁杰等 ${ }^{[12]}$ 前期研究认为, $\mathrm{H}_{6} \mathrm{E}$ 首先与䥻离子螯合形成大小为 $200 \mathrm{~nm}$ 的颗粒, 以此纳米颗粒为模板，在其周围自组装形成表面具 有纳米片状结构的 HAP 微球, 并逐渐形成空心 HAP 微球。

\section{2 粒径与比表面积}

不同 $\mathrm{H}_{6} \mathrm{E}$ 浓度合成条件下, 产物的比表面积由 $8.17 \mathrm{~m}^{2} / \mathrm{g}$ 逐渐增大到 $113.02 \mathrm{~m}^{2} / \mathrm{g}$ 。具体结果见表 1 所示。

表 1 不同 $\mathrm{H}_{6} \mathrm{E}$ 浓度合成条件下产物的比表面积 Table 1 SSA of HAP particles modified by different concentrations of $\mathrm{H}_{6} E$ for synthesis

\begin{tabular}{cc}
\hline Sample & $\mathrm{SSA} /\left(\mathrm{m}^{2} \bullet \mathrm{g}^{-1}\right)$ \\
\hline HAP0 & 8.17 \\
HAP0.5 & 51.83 \\
HAP1 & 65.05 \\
HAP5 & 105.20 \\
HAP50 & 113.02 \\
\hline
\end{tabular}




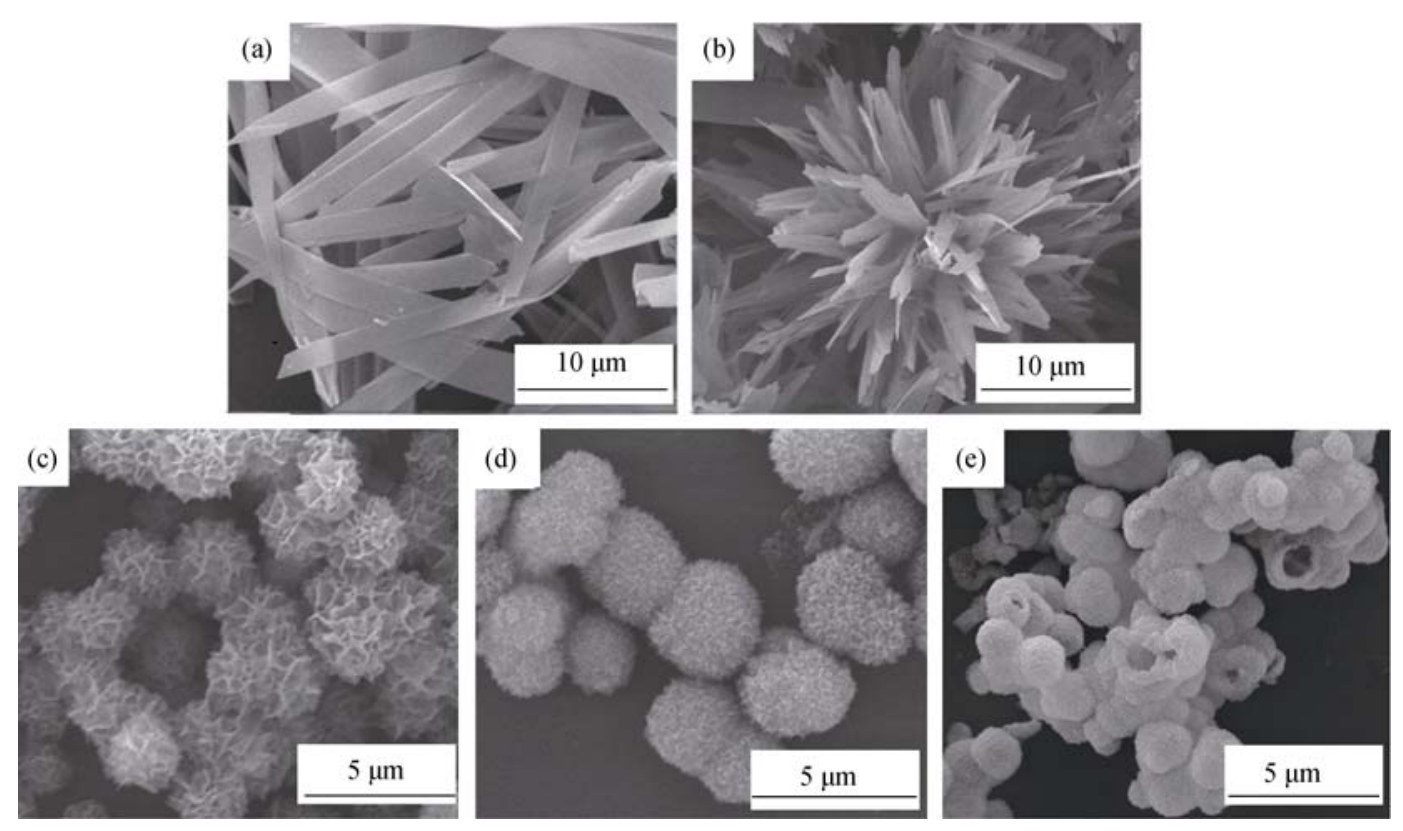

图 1 合成体系中不同浓度 $\mathrm{H}_{6} \mathrm{E}$ 调控下产物的 SEM 照片

Fig. 1 SEM images of products synthesized at various $\mathrm{H}_{6} \mathrm{E}$ concentrations (a) 0 ; (b) $0.5 \mathrm{mmol} / \mathrm{L}$; (c) $1 \mathrm{mmol} / \mathrm{L}$; (d) $5 \mathrm{mmol} / \mathrm{L}$; (e) $50 \mathrm{mmol} / \mathrm{L}$

\section{$2.3 \mathrm{X}$ 射线衍射分析}

与 HAP 标准图谱(JCPDS 09-0432)相比, 不同 浓度 $\mathrm{H}_{6} \mathrm{E}$ 合成条件下, 产物均为 $\mathrm{HAP}$ (图 2), 但是其 结晶度却各有不同。未添加 $\mathrm{H}_{6} \mathrm{E}$ 时, 呈长带状 HAP0 产物(图 1(a))具有较强(300)晶面衍射强度; 随着合 成体系中 $\mathrm{H}_{6} \mathrm{E}$ 浓度增加, 产物中带状结构逐渐缩短 (图 1(b d)), 产物(300)晶面的衍射强度逐渐减弱。 Johnsson 等 ${ }^{[13]}$ 认为某些小分子(柠檬酸三钠、磷酸枸 椽酸盐等)能够选择性地吸附于 HAP 晶面, 选择性 地抑制相应晶面的生长, 对 HAP 的形貌具有调控作 用。Jokić 等 ${ }^{[14]}$ 在研究水热反应条件对 HAP 形貌控 制的实验中发现, 通过调整反应体系的 $\mathrm{pH}$, 可以使

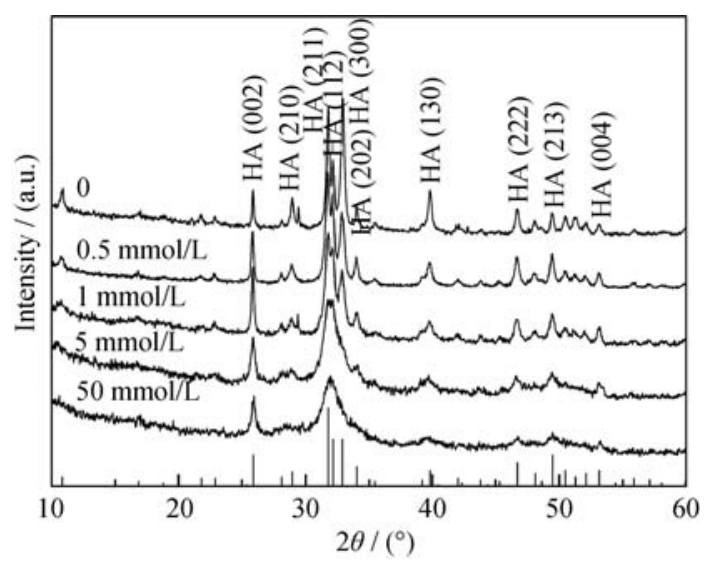

图 2 合成体系中不同浓度 $\mathrm{H}_{6} \mathrm{E}$ 调控下产物的 XRD 图谱

Fig. 2 XRD spectra of products synthesized at various $\mathrm{H}_{6} \mathrm{E}$ concentrations

(a) 0 ; (b) $0.5 \mathrm{mmol} / \mathrm{L}$; (c) $1 \mathrm{mmol} / \mathrm{L}$; (d) $5 \mathrm{mmo} / \mathrm{L}$; (e) $50 \mathrm{mmol} / \mathrm{L}$
HAP 沿着六方晶体 $c$ 轴方向择优生长, 制备出晶须 状的 HAP。可见, HAP 的形貌与小分子晶面吸附导 致的生长抑制和环境参数等因素相关。本实验中, 随着合成体系中 $\mathrm{H}_{6} \mathrm{E}$ 浓度逐渐增大, 产物中带状结 构逐渐缩短, 晶粒逐渐细化, 结晶度逐渐降低。综上 可知, $\mathrm{H}_{6} \mathrm{E}$ 的引入不影响 HAP 的晶相组成, 但显著 地影响 HAP 的晶粒尺寸、晶体生长的取向和产物的 形貌。

\section{4 红外光谱分析}

实验采用红外光谱仪(FTIR, Thermo Nicolet5700) 对 BSA、LYS、FN 在 HAP 颗粒样品上的吸附进行 了检测。图 3 显示了 HAP0 及 HAP0 吸附 BSA、LYS、 $\mathrm{FN}$ 后的红外光谱图。565、603 $\mathrm{cm}^{-1}$ 处为 $\mathrm{PO}_{4}{ }^{3-}$ 的弯 曲振动峰; 960、1033 和 $1111 \mathrm{~cm}^{-1}$ 处 ${ }^{[15]}$ 为 $\mathrm{PO}_{4}{ }^{3-}$ 的伸 缩振动峰。 $873 、 1414$ 和 $1456 \mathrm{~cm}^{-1}$ 处的峰源于 $\mathrm{CO}_{3}{ }^{2-}$ 替换了 $\mathrm{HAP}$ 晶体结构中的 $\mathrm{PO}_{4}{ }^{3-}$ 和 $\mathrm{OH}^{-}$，这说明形成 了 $\mathrm{AB}$ 型含碳 $\mathrm{HAP}^{[15]}$ 。1505 $\mathrm{cm}^{-1}$ 和 $1545 \mathrm{~cm}^{-1}$ 处是 $\mathrm{N}-\mathrm{H}$ 弯曲振动和 $\mathrm{C}-\mathrm{N}$ 伸缩振动峰 ${ }^{[5]}$, 证明了蛋白质的存 在; 同时，吸附蛋白后，HAP 表面基团的吸收峰没有 发生化学位移，这说明 BSA、LYS 和 FN 在 HAP 表 面的吸附以物理吸附为主。

\section{5 蛋白质吸附}

随着合成体系中 $\mathrm{H}_{6} \mathrm{E}$ 的增加, HAP 微粒对 BSA 的吸附性能逐渐减弱(图 4(a))。HAP0 对 BSA 吸附 性能最强, 达 $2.39 \mathrm{mg} / \mathrm{m}^{2}$; HAP50 对 BSA 的吸附性 能最弱, 仅 $0.23 \mathrm{mg} / \mathrm{m}^{2}$ (单个 BSA 分子吸附面积为 


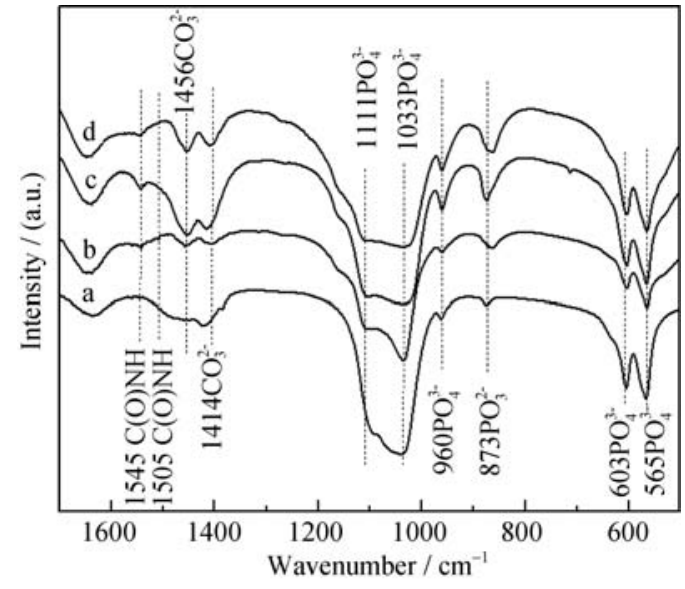

图 3 HAP0 及其吸附不同蛋白质后的 FT-IR 图谱

Fig. 3 FT-IR spectra of (a) HAP0, (b) HAP0-BSA, (c) HAP0LYS and (d) HAP0-FN particles

$14.0 \mathrm{~nm} \times 4.0 \mathrm{~nm}$, BSA 理论最大吸附量为 $2.52 \mathrm{mg} / \mathrm{m}^{2[16]}$ )。 说明 HAP0 微粒单位面积有更多吸附位点; 随着合 成体系中 $\mathrm{H}_{6} \mathrm{E}$ 增加, HAP 表面吸附位点逐渐减少, 蛋白吸附量随之减少。

研究发现 ${ }^{[17-18]}$, HAP 晶体(300)晶面由于富集钙 离子而带正电荷。BSA 等电点为 4.7 , 在 $\mathrm{pH}$ 为 7 时 带负电荷, 因此 HAP 晶体(300)晶面与 BSA 之间存 在静电引力而促进其吸附。在 HAP0(图 1(a))中带状 结构(300)晶面衍射强度最大(图 2), 其单位面积正 电荷最多, 与 BSA 之间静电引力作用最强, 因而对 BSA 的吸附性能最强; 随着合成体系中 $\mathrm{H}_{6} \mathrm{E}$ 逐渐增 加, HAP(300)晶面衍射强度逐渐降低, HAP 微粒表 面正电荷逐渐减少, 与 BSA 之间静电吸引作用逐渐 减弱，因而对 BSA 吸附性能逐渐减弱。

$\mathrm{FN}$ 等电点为 5.5 , 在 $\mathrm{pH}$ 为 7 时带负电荷, $\mathrm{FN}$ 与 HAP 吸附的机制和 BSA 与 HAP 吸附的机制相同。 随着合成体系中 $\mathrm{H}_{6} \mathrm{E}$ 的增加, HAP 微粒对 $\mathrm{FN}$ 的吸 附性能逐渐减弱(图 4(b))。HAP0 对 FN 的吸附性能 最强, 达 $5.41 \mathrm{mg} / \mathrm{m}^{2}$; HAP50 对 $\mathrm{FN}$ 的吸附性能最弱, 仅为 $0.41 \mathrm{mg} / \mathrm{m}^{2}$ 。

然而，具有不同表面微纳结构的 HAP 微粒对 LYS 表现出不同于 BSA、FN 的吸附行为, 随着合成体系中 $\mathrm{H}_{6} \mathrm{E}$ 的增加, HAP 微粒对 LYS 的吸附性能逐渐增强(图 4(c))。HAP0 对 LYS 的吸附性能最弱, 仅 $0.32 \mathrm{mg} / \mathrm{m}^{2}$; HAP50 对 LYS 的吸附性能最强, 达 $0.86 \mathrm{mg} / \mathrm{m}^{2}$ 。(单个 LYS 分子吸附面积为 $3.0 \mathrm{~nm} \times 4.5 \mathrm{~nm}, \mathrm{LYS}$ 在材料表 面理论最大吸附量为 $2.02 \mathrm{mg} / \mathrm{m}^{2[16]}$ )。说明 HAP50 微粒单位面积具有更多吸附位点; 随着合成体系中 $\mathrm{H}_{6} \mathrm{E}$ 的增加, HAP 表面吸附位点逐渐增加, 蛋白吸 附量随之增加。

另外, LYS 的等电点在 11.0 11.35 之间, 在 $\mathrm{pH}$
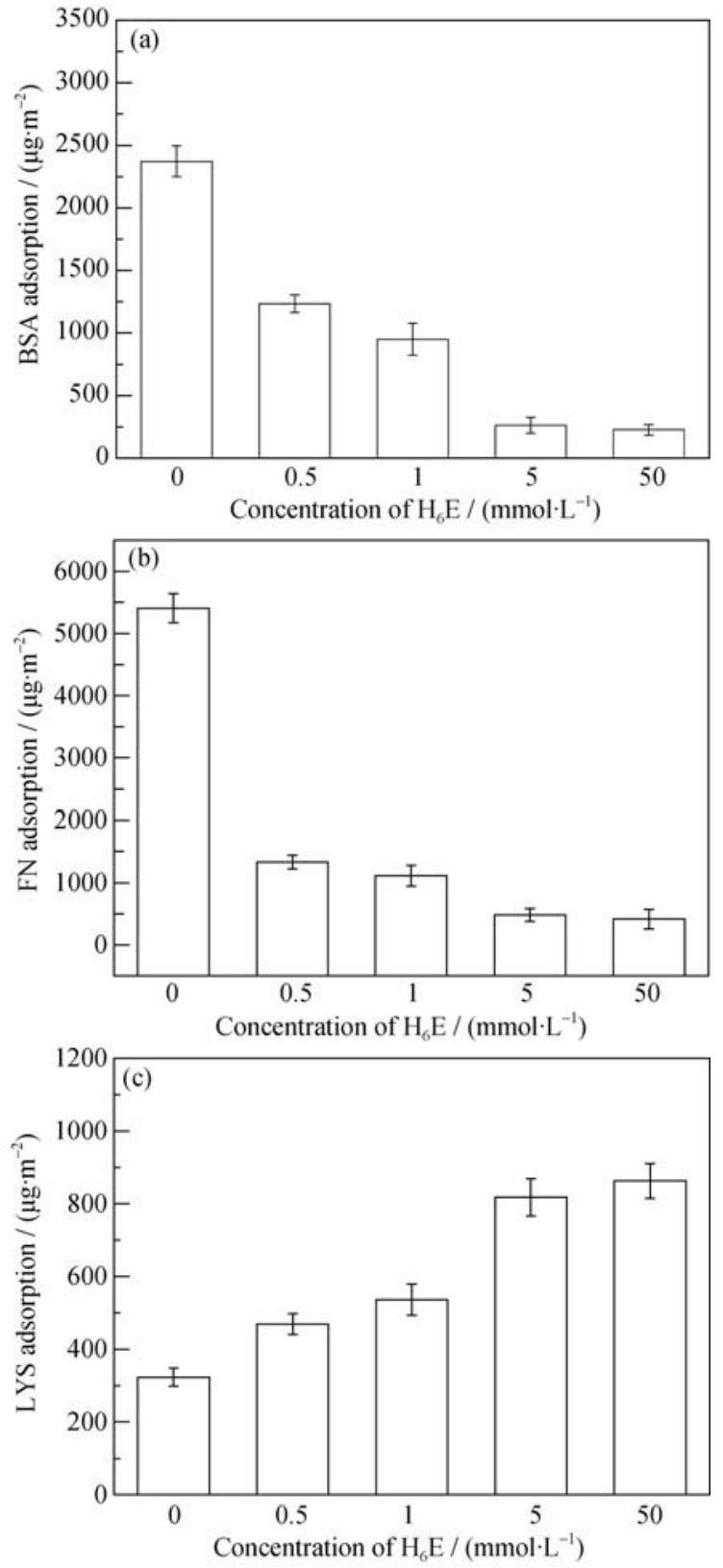

图 4 三种蛋白质在不同表面形貌的 HAP 表面的吸附行为 Fig. 4 The adsorption properties between HAP particles with different surface morphologies and different kind of proteins (a) BSA; (b) FN; (c) LYS $(p<0.05)$

为 7 时带正电荷, 因此 HAP 晶体(300)晶面与 LYS 之间存在静电斥力而抑制其吸附。HAP0(图 1(a))的 长带状结构(300)晶面衍射强度最大(图 2), 单位面 积正电荷最多，与 LYS 分子之间静电斥力作用最强, 对 LYS 的吸附性能最弱。随着合成体系中 $\mathrm{H}_{6} \mathrm{E}$ 增加, $\mathrm{HAP}(300)$ 晶面衍射强度逐渐降低，单位面积正电荷 逐渐减少, 与 LYS 之间静电斥力逐渐减弱, 对 LYS 的吸附增大。

如图 5 所示, 单位质量的 HAP0、HAP50 颗粒 对 BSA 和 FN 的吸附总量相近, 而单位质量 HAP50 对 LYS 的吸附总量是单位质量 HAP0 对 LYS 吸附 


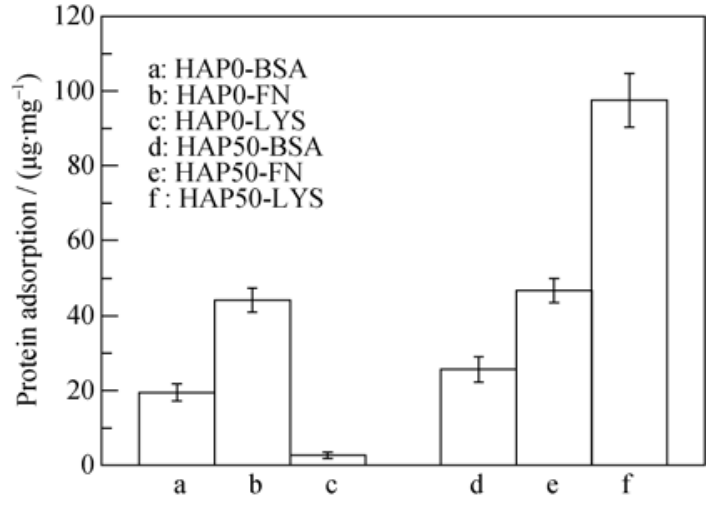

图 5 单位质量 HAP0 和 HAP50 对不同蛋白质的吸附行为

Fig. 5 Adsorption behavior of different proteins on unit mass of HAP0 and HAP50 samples $(p<0.05)$

总量的 37 倍(图 5-c、图 5-f)。说明, HAP50 的中空 结构对碱性蛋白质具有较大的吸附性能。

综上可知, HAP 颗粒对蛋白质的吸附量并不仅 仅随着 HAP 颗粒比表面积(表 1)增大而增大，而与 HAP 微粒表面的晶面结构具有直接关系。

\section{6 蛋白质释放}

蛋白质在 HAP 微粒上吸附后的体外蛋白质释放 实验结果表明: HAP0-BSA、HAP0-FN 和 HAP0-LYS 具有明显突释行为, 前 $5 \mathrm{~h}$ 蛋白释放量达到 $75 \%$ 以上, $24 \mathrm{~h}$ 释放量达到 $95 \%$ 以上; 载蛋白 HAP0.5、HAP1 和 HAP5 微粒表现出逐渐增强的蛋白缓释性能, 前 $5 \mathrm{~h}$ 释放量约 $60 \%$, 蛋白释放速率逐渐降低; 载蛋白 HAP50 微粒缓释效果最明显，前 $5 \mathrm{~h}$ 释放量在 $45 \%$ 左 右, 在 $5 \mathrm{~h}$ 到 $168 \mathrm{~h}$ 范围内呈现缓慢释放的趋势。这 说明中空壳层结构 HAP50 能使释放速度降低, 释放 时间延长(图 6(a c)。鉴于红外光谱结果(图 3), 蛋白 与 HAP 之间以物理吸附为主, 所以 HAP 微粒上蛋 白释放行为主要决定于其表面的微纳结构。

Wang 等 ${ }^{[19]}$ 用水热法制备 HAP 球粒, 载布洛芬 HAP 球粒释放试验表明: 表面光滑 HAP 突释现象严重, 而烟花状 HAP 具有明显布洛芬缓释性能, 认为 HAP 表 面粗粘和多孔结构能有效减缓布洛芬的释放速度。

Sun 等 ${ }^{[20]}$ 用喷雾干燥法制备 HAP 中空球粒，载 胰岛素 HAP 球粒体外释放实验表明：中空球粒 HAP 对胰岛素具有缓释作用。在本实验中, 由于空 间位阻 ${ }^{[21]}$ 、机械锁合等作用, 表面粗糙的 HAP 与蛋 白质之间结合力较强, 因此蛋白释放速度较慢; 相 反, 光滑表面 HAP 微粒与蛋白质分子间结合力较弱, 蛋白突释现象严重。

以上结果表明, HAP 微粒表面形貌能明显地调 控其表面吸附蛋白质的释放速率。然而 HAP 表面形 貌与晶粒尺寸、结晶度、微孔隙等诸多因素有关，具
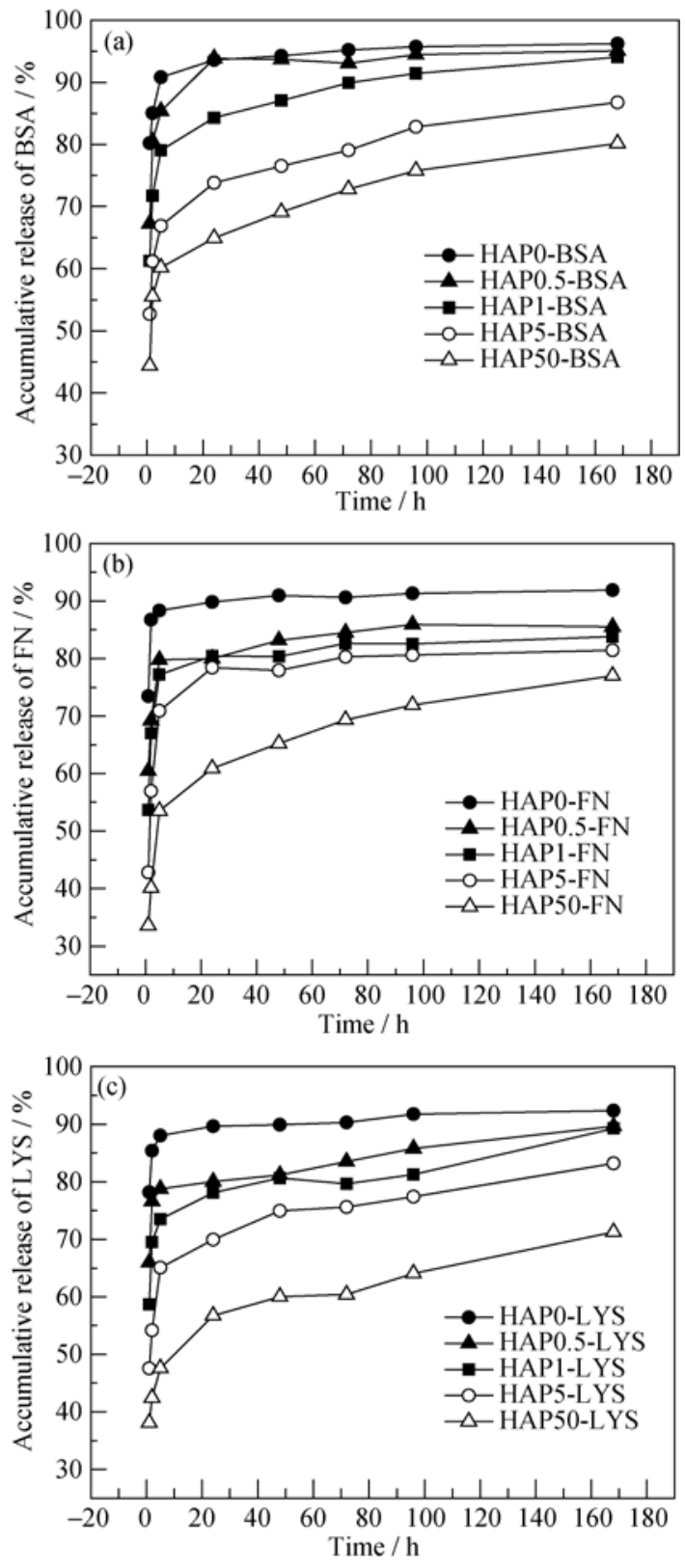

图 6 具有不同表面微纳结构 HAP 载三种蛋白体外释放曲线 Fig. 6 The release curves of (a) BSA, (b) FN and (c) LYS from protein-loaded HAP particles with different surface morphologies

体导致 HAP 表现出不同蛋白缓释行为的因素还有 待进一步研究。

\section{3 结论}

本研究采用水热法，以不同浓度的 $\mathrm{H}_{6} \mathrm{E}$ 作为模 板，制备具有不同表面微纳结构的 HAP 微粒，并研 究了 HAP 表面微纳结构对 BSA、FN、LYS 吸附及 释放的作用规律。研究发现:

1) 随着合成体系中 $\mathrm{H}_{6} \mathrm{E}$ 浓度增加, HAP 晶体生 长取向发生变化, (300)晶面衍射强度逐渐降低。 
2) HAP 表面微纳结构对蛋白质具有选择性吸附 作用: 带状结构有利于酸性蛋白质(BSA 和 FN)的吸 附，但其单位质量对 BSA 和 FN 的吸附与 HAP50 的 吸附总量相近, 并且其蛋白质体外释放存在明显的 突释现象。

3) 中空结构 HAP(HAP50)对 LYS 吸附性能最强, 并且其载蛋白后具有明显的体外蛋白缓释性能。

4) 这些实验结果对研究 HAP 表面不同微纳结 构与蛋白质吸附一释放之间的相互关系提供了有益 的借鉴。

\section{参考文献:}

[1] MASKARINEC S A, TIRRELL D A. Protein engineering approaches to biomaterials design. Current Opinion in Biotechnology, 2005, 16(4): 422-426.

[2] DUCHEYNE P, QIU Q. Bioactive ceramics: the effect of surface reactivity on bone formation and bone cell function. Biomaterials, 1999, 20(23/24): 2287-2303.

[3] LIAO X L, LU S Y, ZHUO Y, et al. Bone physiology, biomaterial and the effect of mechanical/physical microenvironment on mesenchymal stem cell osteogenesis. Cellular and Molecular Bioengineering, 2011, 4(4): 579-590.

[4] YOSHIMOTO H, SHIN Y M, TERAI H, et al. A biodegradable nanofiber scaffold by electrospinning and its potential for bone tissue engineering. Biomaterials, 2003, 24(12): 2077-2082.

[5] ZHANG H J , ZHU X D, WANG X L, et al. Effect of sintering processes on surface properties and protein adsorption of hydroxyapatite ceramic particles. Journal of Inorganic Materials, 2010, 25(7): 771-774.

[6] SANTOS E A, FARINA M, SOARES G A, et al. Surface energy of hydroxyapatite and $\beta$-tricalcium phosphate ceramics driving serum protein adsorptionand osteoblast adhesion. Mater. Sci. Mater. Med., 2008, 19: 2307-2316.

[7] ROUAHI M, CHAMPION E, GALlET O, et al. Physico-chemical characteristics and protein adsorption potential of hydroxyapatite particles: influence on biocompatibility of ceramics after sintering. Colloids and Surfaces B: Biointerfaces, 2006, 47(1): 10-19.

[8] YUAN H P, YANG Z J, LI Y B, et al. Osteoinduction by calcium phosphate biomaterials. Materials in Medicine, 1998, 9(12): 723-726.

[9] LORD M S, FOSS M, BESENBACHER F. Influence of nanoscale surface topography on protein adsorption and cellular response. Nanotoday February, 2010, 5(1): 66-78.

[10] FUJII E, OHKUBO M, TSURU K, et al. Selective protein adsorption property and characterization of nano-crystalline zinc-containing hydroxyapatite. Acta Biomater. 2006, 2(1): 69-74.

[11] ELANGOVAN S, MARGOLIS H C, OPPENHEIM F G, et al. Conformational changes in salivary proline-rich protein 1 upon adsorption to calcium phosphate crystals. Langmuir, 2007, 23(22): $11200-11205$.

[12] XIAO D Q, SHI F, YAO N, et al. Nanostructured hydroxyapatite microspheres by templated synthesis: formation and mechanisms. Progress in Biochemistry and Biophysics, 2013, 40(10): 935-947.

[13] JOHNSON M, RICHARDSON C F, SALLIS J D, et al. Adsorption and mineralization effects of citrate and phosphocitrate on hydroxyapatite. Calcified Tissue International, 1991, 49(2): 134-137.

[14] JOKIć B, MITRIć M, RADMILOVIć V, et al. Synthesis and characterization of monetite and hydroxyapatite whiskers obtained by a hydrothermal method. Ceramics International, 2011, 37(1): 167-173.

[15] KOUTSOPOULOS S. Synthesis and characterization of hydroxyapatite crystals: a review study on the analytical methods. Journal of Biomedical Materials Research, 2002, 62(4): 600-612.

[16] ARAI T, NORDE W. The behavior of some model proteins at solid-liquid interfaces 1 . adsorption from single protein solutions. Colloids and Surface, 1990, 51: 1-15.

[17] KANDORI K, SHIMIZU T, YASUKAWA A, et al. Adsorption of bovine serum albumin onto synthetic calcium hydroxyapatite: influence of particle texture. Biointerfaces, 1995, 5(1/2): 81-87.

[18] KANDORI K, FUDO A, ISHIKAWA T. Study on the paritcle texture dependence of protein adsorption by using synthetic micrometer-sized calcium hydroxyapatite particles. Colloids and Surfaces B: Biointerfaces, 2002, 24(2): 145-153.

[19] WANG Y S, HASSAN M S, GUNAWAN P, et al. Polyelectroly temediated formation of hydroxyapatite microspheres of controlled size and hierarchical structure. Journal of Colloid and Interface Science, 2009, 339(1): 69-77.

[20] SUN R X, CHEN K Z, LU Y P. Fabrication and dissolution behavior of hollow hydroxyapatite microspheres intended for controlled drug release. Materials Research Bulletin, 2009, 44(10): 1939-1942.

[21] AMOOZGAR Z, YEO Y. Recent advances in stealth coating of nanoparticle drug delivery systems. Nanomedicine and Nanobiotechnology, 2012, 4(2): 219-233. 